\title{
Textos básicos sobre el estado de Guerrero
}

Carlos Illades

La bibliografía referente al estado de Guerrero no es tan extensa como la que se ocupa de otros estados de la República. Este hecho resulta singular si se toma en cuenta, por ejemplo, la ubicación geográfica de Guerrero o que muchos acontecimientos de alcance nacional se desarrollaron en la entidad suriana; tal es el caso de la guerra de Independencia o de la rebelión de Juan $\mathrm{N}$. Alvarez en contra del gobierno de Santa Anna.

Una posible causa de esta relativa carencia de materiales bibliográficos sobre el estado de Guerrero - aunque por supuesto no la única - la constituý en la segunda mitad del siglo XIX y parte del XX la ausencia de una intelectualidad guerrerense más o menos amplia que se ocupara de estas cuestiones y, en las últimas décadas, la falta de centros encargados de reunir, sistematizar y analizar la información sobre la entidad. A este respecto habría que señalar que el monumental trabajo realizado por Alejandro W. Paucic y la producción historiográfica de Moisés Ochoa Campos constituyen una excepción, ya que se trata de investigaciones realizadas de manera autónoma y, en el caso de Paucic, por un extranjero.

En años recientes se han subsanado algunas de las lagunas de información sobre el estado sureño, gracias a los trabajos emprendidos por la Universidad Autónoma de Guerrero y, últimamente, por el Instituto Guerrerense de la Cultura. No obstante los esfuerzos realizados por ambas instituciones y por investigadores independientes, el conocimiento que tenemos de la entidad es aún precario.

Muchos de los estudios publicados sobre el estado de Guerrero pertenecen al campo de la historiografía. Dentro de éste han predominado dos enfoques: el enciclopédico y el apologético. En el primer caso, la narración de los hechos históricos se incluye dentro de obras generales que se ocupan de la geografía, economía, administración pública, división municipal, costumbres y tradiciones, monografías, etc., de la entidad suriana. En estos textos, huelga decirlo, el tratamiento del acontecer histórico es más bien descriptivo y no se pone atención en los aspectos explicativos o causales. No obstante esta limitación, con este enfoque se elaboraron obras de gran valor como el Diccionario geográfico, histórico y lingüístico del estado de Guerrero (1942) del general Héctor F. López o la Geografia del estado de Guerrero y síntesis histórica (1959) de Amado González Dávila.

La historiografía de corte apologético ha resultado particularmente prolija, teniendo como género predilecto el biográfico. Su leit motiv lo constituyeron las celebraciones locales y nacionales y, por ende, las fechas relevantes. Buen ejemplo dentro de este campo lo constituye gran parte de la obra de Moisés Ochoa Campos, aunque, justo es reconocerlo, varios de sus trabajos se salen de este marco y constituyen puntos de referencia obligados para el estudio general de la historia del estado de Guerrero, como es el caso de su Historia del estado de Guerrero (1968). 
Durante los últimos años, historiadores profesionales emprendieron el estudio de la entidad suriana. El signo característico de sus trabajos lo constituyó la especialización. Así, Edgar Pavía elaboró diversos trabajos sobre la historia colonial, en particular sobre el siglo XVI, Jaime Salazar sobre el Porfiriato y José Manuel López Victoria se abocó a estudiar la Revolución de 1910. En estas investigaciones, así como en las de otros historiadores contemporáneos (Alvaro López Miramontes, Marcelo González Bustos, etc.), cobró vigor el estudio de la historia social, relegada en el pasado en favor de la historia política, con el consecuente énfasis en la historia vista como proceso y no sólo en las fechas o acontecimientos puntuales.

La geografía y economía guerrerenses también han sido estudiadas de manera más o menos amplia. En lo referente a la primera, necesariamente hay que hacer mención del archivo que reunió a lo largo de su vida el ingeniero yugoslavo - italiano, según algunos - Alejandro Wladimir Paucic Smerdu. Desde 1933 hasta el momento de su muerte, ocurrida en 1980, el ingeniero Paucic recopiló información muy variada (geográfica, histórica, etnológica, lingüística, etc.) y, en especial, sobre la geografía guerrerense. Lamentablemente, sólo una pequeña parte de su obra ha sido publicada, conservándose parte de su archivo en el Instituto Guerrerense de la Cultura ubicado en Acapulco. En 1980, Esperanza Figueroa publicó el Atlas geográfico e histórico del estado de Guerrero, apoyándose íntegramente en la información reunida por Paucic.

En el campo de la economía destacan el trabajo sobre la costa de Guerrero elaborado por Benjamín Retchkiman (1948) y el texto ya clásico de Moisés T. Peña titulado Guerrero económico (1949), en el cual colaboró el propio Retchkiman. La Universidad Autónoma de Guerrero, en años recientes, ha impulsado el estudio de la economía regional; entre sus publicaciones sobre la materia destaca el libro de Manuel Torres, Luis Méndez y Jorge Arcila (La estructura económica del estado de Guerrero, 1983).

Menos atención han ocupado los temas de carácter antropológico y sociológico. En estos campos es relevante la obra de Gonzalo Aguirre Beltrán (Cuijla, 1958) sobre la población negra de Cuajinicuilapa, Guerrero y las diversas investigaciones desarrolladas por Francisco A. Gomezjara.

- La bibliografía comentada que presentamos está agrupada en trece temas, a saber: historia (subdividido en: obras generales, siglo XIX y siglo XX), biografías, memorias, geografía, economía, antropología, problemas agrarios, viajeros y cronistas, folklore, monografías, literatura, legislación y bibliografías y guías documentales. Aunque muchos textos en sentido estricto podrían entrar en más de un rubro, con la intención de facilitar al lector su consulta hemos decidido incorporarlos al apartado sobre el cual brindan mayor información.

Confiamos en que esta bibliografía sirva de base para quienes se inician en el estudio de la realidad guerrerense.

\section{HISTORIA (OBRAS GENERALES)}

BNAL

040.37

ENSNG. 11 1974
Chávez Reyes, María Guadalupe, Estudio sintético de lugares y personajes historicos en el estado de Guerrero, Guadalajara, Escuela Normal Superior Nueva Galicia, 1974, 173 p. (tesis). 
Trabajo en el cual se evalúa la importancia del estudio de la historia del estado de Guerrero para los alumnos de nivel medio. Se hace mención de los lugares donde ocurrieron hechos historicos fundamentales (Iguala, Chilpancingo, Taxco, Tixtla, Chilapa, Tecpan y Acapulco) y de los personajes que trascendieron el ámbito regional (Vicente Guerrero, Hermenegildo Galeana, Juan N. Alvarez, Ignacio Manuel Altamirano y la familia Bravo). No se hace una revisión crítica ni de los hechos históricos ni de los personajes que intervinieron en ellos; no obstante, la obra representa un intento por abordar la historia con fines didácticos.

BNAL

972.71

Gue. s

Guevara Ramírez, Luis, Síntesis histórica del estado de Guerrero, México, Gráfica Cervantina, 1959, 126 p. (Colección de Estudios Históricos Guerrerenses, 1).

Apretada síntesis de la historia de la entidad, desde los tiempos prehispánicos hasta la Revolución de 1910. La guerra de Independencia, la erección del estado en 1849 y la revolución de Ayutla ocupan en mayor medida el interés del autor. Como en casi toda la historiografía guerrerense, en esta obra se pone énfasis en la historia política, dando un espacio menor a los aspectos económicos, sociales e ideológicos. En este trabajo predomina la descripción sobre el análisis. Al final se anexa una bibliografía básica sobre la historia de la entidad suriana.

BIM

972.73

Ens. $p$

BPCECH

Lopez Miramontes, Alvaro et al., Ensayos para la historia del estado de Guerrero, prol. Francisco Pérez Arce, Chilpancingo, Instituto Guerrerense de la Cultura, 1985, 222 s.c. p., ils.

Los once ensayos que componen el texto proporcionan abundante y variada información sobre la historia del estado de Guerrero. Alvaro López Miramontes realiza una rápida revisión de la producción historiográfica dedicada a la entidad, a la vez que Roberto Cervantes-Delgado hace un recuento sumario de las crónicas de los viajeros que han visitado el estado. Al lado de los ensayos de Nicole Girón ("Ignacio Manuel Altamirano en la vida política del estado de Guerrero"), Gerald L. McGowan ("Alvarez y Santa Anna ante la prensa"), Ignacio Martínez Rivera y Gabino Olea Campos ("La revolución en la lucha agraria de la Costa Grande") destacan los trabajos de Rogelio Vizcaíno y Paco Ignacio Taibo II ("El socialismo en un solo puerto") sobre el movimiento escuderista en Acapulco y el de Cayetano Reyes ("El jefe agrarista costeño Valente de la Cruz") sobre el revolucionario nacido en Tecpan de Galeana. Un anexo formado por tres pequeños ensayos complementa el texto. México, Librería de Porrúa Hnos. y Cía. S.A.,1968, 375 p., ils. $\mathrm{y}$ fotos.

BNAL

972.71

El autor, que al ser publicada la obra era presidente de

Och.h

la Sección de Historia de la Sociedad Mexicana de Geografía y Estadística, comienza por informar acerca del medio geográfico y situación económica de la entidad, para después abordar su desarrollo histórico. La obra consta de cinco partes: I. El sur hasta 1808; II. De la Independencia hasta la dictadura de Santa Anna; III. El Plan de Ayutla, la revolución de Reforma y la guerra contra la intervención; IV. El sur durante el Porfiriato, y V. La Revolución mexicana. Contiene tres apéndices (historia de la cultura, gobernantes y calenda- 
rio de efemérides guerrerenses). Aunque cae en los lugares comunes de la historiografía dominante: apología de los liberales, del constitucionalismo y de los gobiernos posrevolucionarios, el texto de Ochoa Campos constituye, a la fecha, la historia general de Guerrero más amplia y completa.

CONDUMEX 079:7271

Ort.

A. No. 17590-C

BIM

079.7273

Orti

Ortega, Miguel F., La imprenta y el periodismo en el sur. Homenaje a la intelectualidad guerrerense, México, Ediciones Pluma y Lápiz de México, 1943, 58 p.

En este opúsculo, el autor sigue el desarrollo de la prensa en el actual estado de Guerrero desde finales de la época colonial hasta los últimos años del siglo XIX. En cada uno de los momentos históricos relevantes se señala cuál periódico tuvo mayor influencia en la opinión pública local. Al final de texto, se presenta un listado de los intelectuales guerrerenses más destacados del siglo XIX, agrupándolos de acuerdo con su actividad profesional.

\section{HISTORLA (siglo XIX)}

BNAL

972.71

Car. a

BCRA

G

972.73

Car. a

Carranco Cardoso, Leopoldo, Acciones militares en el estado de Guerrero, México, Sociedad Mexicana de Geografía y Estadística, 1963, 95 p.

Narración de los conflictos armados más importantes en la entidad desde la consumación de la Independencia hasta la intervención francesa. El autor realiza una apología de la figura de Juan $\mathrm{N}$. Alvarez y destaca la similitud del pensamiento de éste con el de Benito Juárez. Reseña los acontecimientos motivados por el sitio y toma de Taxco por parte de Porfirio Díaz en 1863 y describe la vida social en Acapulco durante la intervención francesa. A lo largo de la obra se pone de relieve al patriotismo del pueblo guerrerense y sus caudillos.

COLmeX Díaz Díaz, Fernando, Caudillos y caciques, México, El Co-

92

D542lc

1972 legio de México/Centro de Estudios Históricos, 1972, 354

p. (Nueva Serie, 15).

El autor, colombiano, doctor en historia por El Colegio de México, estudia con ayuda de las categorías de la sociología weberiana la acción política de Antonio López de Santa Anna (el caudi1lo) y Juan N. Alvarez (el cacique). A la vez que sigue paso a paso las pugnas entre ambos personajes, revisa la historia de México desde 1810 hasta los comienzos del Porfiriato. Este trabajo constituye uno de los pioneros sobre el cacicazgo de Juan N. Alvarez y sobre las estructuras del poder político en México durante el siglo XIX.

BNAL Domínguez, Miguel, La erección del estado de Guerrero.

972.71

Dom. $\theta$ Antecedentes históricos, México, SEP, 1949, 108 p., ils. y

BPCECH facs.

s.c.

Texto de la ponencia presentada ante el Congreso Mexicano de Historia, con motivo del centenario de la erección del estado de Guerrero. El autor se remonta a la guerra de Independencia para explicar cómo se generó la necesidad de crear una entidad federativa en el sur. Se narran los conflictos en los estados afectados por la formación de Guerrero. En la parte final, se incorpora 
un apénđice con la documentación más relevante referida a la erección de la entidad suriana.

BPCECH Guerrero Gómez, Gerardo y Santano González Villalos.c. bos, Personajes del estado de Guerrero. Su aportación a la historia nacional, Chilpancingo, Instituto Guerreren44 p., ils. se de la Cultura/Gobierno del estado de Guerrero, 1987,

Texto dirigido a la nif́ez, elaborado en forma de historieta, sobre la participación de Nicolás Bravo y Vicente Guerrero en la lucha por la Independencia nacional. No obstante el maniqueísmo y simplismo del que adolecen por lo general los libros de historia dirigidos a los niños, el texto posee información de utilidad sobre el proceso de independencia en el estado.

BEEUAG Salazar Adame, Jaime, Movimientos populares durante el s.c. Porfiriato en el estado de Guerrero, Chilpancingo, Univer-

BIM

972.00

Mis. 11 sidad Autónoma de Guerrero, 1983, 73 p. (Serie Historia de Guerrero, 2).

Análisis histórico de la formación de los cacicazgos en Guerrero durante el último cuarto del siglo XIX. Aunque estudia varios movimientos, en especial indaga sobre la rebelion del general Canuto A. Neri en 1891, que tuvo como objetivo central impedir la cuarta reelección como gobernador de Guerrero del general zacatecano Francisco O. Arce. El autor, guerrerense, formado en la Universidad Iberoamericana, es en la actualidad el principal estudioso del Porfiriato en la entidad.

BNAL Velasco, Emilio, Discurso pronunciado en la sesión del 4 308 del corriente sobre la cuestión de Guerrero, México, ImMis. 132 prenta de I. Cumplido, 1871, $16 \mathrm{p}$.

El 26 de mayo de 1870, cl general zacatecano Francisco O. Arce fue desconocido como gobernador por la Legislatura del estado de Guerrero, pero regresó a su cargo con el apoyo del gobierno federal y gracias a la intervención del ejército. El Congreso de la Unión, en el que figuraba como diputado el autor del texto que comentamos, considero justa y acertada la decisión de la Legislatura guerrerense; por tal motivo, entró en abierto conflicto en el Ejecutivo, encabezado por Benito Juárez. Por otra parte, ante la intervención del ejército federal, el general Vicente Jiménez se declaró en rebeldía contra los gobiernos federal y estatal. Al poco tiempo la rebelión de Jiménez fue sofocada. Este discurso de Emilio Velasco, es un fuerte alegato en contra del general Arce y de la intervención del gobierno federal en los asuntos internos del estado de Guerrero.

\section{HISTORLA (siglo XX)}

BNAL Fuentes Díaz, Vicente, La revolución de 1910 en el estado REV 97271.09 Fue. $r$ de Guerrero, México, Nacional Impresora, 1960, 183 p., fotos.

Se comienza por estudiar los movimientos sociales durante el Porfiriato, para después abordar el impacto que tuvieron en Guerrero la revolución maderista y el huertismo. Por otra parte, se analizan las causas económicas y políticas que motivaron la explosión de la 
lucha armada y las características sociales y culturales de los caudillos que dirigieron la revolución de 1910 en Guerrero. Este texto, publicado hace veintisiete años, constituye uno de los "clásicos" sobre el periodo revolucionario en la entidad.

BEEUAG González Bustos, Marcelo, El general Jesús H. Salgado y s.c. el movimiento zapatista en Guerrero, Chilpancingo, UniBIM versidad Autónoma de Guerrero, 1983, 165 p. (Serie 923.672 Grandes Personajes de Guerrero, 3).

Sal.g Tesis presentada en el Colegio de Historia de la UNAM, dirigida por Andrea Sánchez Quintanar, y que obtuvo mención honorífica en el Primer Concurso de Ensayo sobre Historia de Guerrero. En ella, el autor combate la idea según la cual el zapatismo fue tan sólo un movimiento marginal en el estado de Guerrero. Demuestra cómo el general Jesús $H$. Salgado fue un zapatista convencido a lo largo de su vida, truncada violentamente por el coronel Rogelio Flores en el año de 1920. Quizá el valor fundamental de la obra radica en el rescate de la historia, por lo demás poco conocida, de los que perdieron -en tanto que grupos sociales- la revolución en Guerrero.

BIM

972.061

Cau. $y$

Jacobs, Ian, "Rancheros en Guerrero: los hermanos Figueroa y la Revolución", en David A. Brading (comp.), Caudillos y campesinos en la Revolución mexicana, trad. Carlos Valdés, México, Fondo de Cultura Económica, 1985, 336 p., p. 106-124.

El autor, inglés, especialista en la revolución de 1910 en el estado de Guerrero, intenta esclarecer el papel desempeñado en aquellos años por la familia Figueroa, originaria del pueblo de Huitzuco. Sin hacer concesiones a la historiografía oficial, Jacobs sigue de cerca el proceso de conformación del cacicazgo de los Figueroa, para después analizar su participación en la lucha armada al lado de Madero y en contra del movimiento zapatista. Este trabajo constituye, sin duda, una valiosa aportación al estudio de las formas de dominación política dentro del ámbito regional.

COLMEX 97271 JI7r

Jacobs, Ian, Ranchero Revolt. The Mexican Revolution in Guerrero, Texas, University of Texas, 1982, 234 p.

Tesis doctoral sobre la formación y consolidación del poder económico y político de la familia Figueroa. Se estudian las características económicas y sociales del norte guerrerense para después abordar el tema de las haciendas. Por otra parte, se analizan los principales movimientos políticos en el estado en contra de Porfirio Díaz y se señalan los rasgos que caracterizaron a la revolución de 1910 en la entidad. Un capítulo está dedicado a la reforma agraria durante el periodo 1919-1940. El autor destaca la adaptabilidad de la familia Figueroa a la cambiante situación política nacional.

BIM

972.73

Lop. $\mathrm{H}$

BPCECH

s. $c$.

López Victoria, José Manuel, Historia de la revolución en Guerrero, Chilpancingo, Instituto Guerrerense de la Cultura/Gobierno del estado de Guerrero, 1985, 3 vols.

Pormenorizada historia de Guerrero elaborada por un investigador nacido en la entidad. Este amplio trabajo sobre el periodo 1901-1929 vincula de manera estrecha el acontecer nacional con el de la región; así, se da razón del maderismo, 
zapatismo, huertismo, carrancismo, delahuertismo, obregonismo y callismo en la entidad. La obra incorpora muchas fuentes primarias, en especial provenientes de la Secretaría de la Defensa Nacional, y quizá su mérito radica, más que en el análisis, en la narración detallada de los acontecimientos. Como complemento a esta obra, próximamente el autor publicará un diccionario de revolucionarios guerrerenses.

BNAL Valverde, Custodio, Julián Blanco y la revolución en el esREV 972.09:92 Val. j tado de Guerrero, México, Imprenta de J. Chávez y Hno., $1916,216 \mathrm{p}$.

Trabajo de gran importancia sobre la revolución de 1910 en la entidad. El autor, diputado al Congreso local en 1912, realiza una vigorosa defensa del maderismo y del constitucionalismo, en oposición a los movimientos encabezados por Villa y Zapata. En especial, se narran las acciones armadas y se proporciona información sobre sus principales actores. Para Custodio Valverde, Julián Blanco fue el principal protagonista de la revolución de 1910 en Guerrero. Al final de la obra abunda sobre sus méritos y acerca de las causas que motivaron su asesinato - a manos de los seguidores de Silvestre Mariscal-en 1915.

\section{BIOGRAFIAS}

BCRA

G

923.5

Alv. $g$

Alvear Acevedo, Carlos, Galeana, México, Editorial Jus, 1958, 128 p. (Figuras y Episodios de la Historia de México, 48).

Apología de Hermenegildo Galeana y de su participación en la guerra de Independencia al lado de Morelos. El texto consta de cinco capítulos, en los cuales el autor narra las cuatro campañas de Morelos y describe a grandes rasgos los hechos sobresalientes de la revolución de independencia en el territorio del actual estado de Guerrero. Sin pretensiones analíticas, esta biografía consigna información de interés sobre la geografia y vida social de la región al comenzar el siglo XIX.

BPCECH Chávez Guerrero, Herminio, Ignacio Manuel Altamirano, s.c. Chilpancingo, Instituto Guerrerense de la Cultura, 1985, 253 p., ils.

Texto escrito por un connotado biógrafo de la entidad, merecedor del segundo premio del Concurso Nacional de Ensayo Biográfico sobre Ignacio Manuel Altamirano, celebrado en 1984. El autor comienza por apuntar los datos biográficos básicos del escritor nacido en Tixtla, para después ocuparse de su formación intelectual en el Colegio de Toluca y el Colegio de Letrán. Describe el entorno político y militar en que se mueve Altamirano - guerra de Tres Años, imperio de Maximilianoabordando a continuación su producción intelectual, centrada en diversos géneros literarios y en el periodismo.

BIM

923.672 Gom. $m$
Gomezjara, Francisco A., María de la O y Benita Galeana: precursoras del feminismo socialista en Guerrero, Chilpancingo, Universidad Autónoma de Gúerrero, 1982, 68 p., retrs.

Desde una perspectiva sociológica, el autor analiza la militancia comunista de María de la O y Benita Galeana. Con tal fin, presenta a gran- 
des rasgos las características del cardenismo en Guerrero, años de formación política de ambas luchadoras socialistas, así como una breve historia social del estado durante el periodo 1930-1960. Se arroja luz sobre las pugnas al interior del Partido Comunista Mexicano y acerca de la incorporación de María de la $\mathrm{O}$ a la Federación de Partidos del Pueblo Mexicano. El autor, investigador de la UNAM, ha dedicado buena parte de su obra al estudio del estado de Guerrero.

BIM

086

Bep. 124

Lafragua, José María, Vicente Guerrero: el mártir de Cuilapam, arreglo y notas de Fernando Iturribarría, México, SEP, 1946, 94 p., retrs. (Biblioteca Enciclopédica Popular, 124).

Texto escrito por el secretario de Relaciones Exteriores del gobierno de Benito Juárez, quien pretendio sentar las bases sobre las que pudiera “...levantarse la historia del héroe del Sur, cuando las pasiones y los intereses de partido, hoy ya bastante debilitados, hayan desaparecido completamente, cediendo su puesto a la justicia y a la verdad". No obstante pretender contar lo "bueno" y lo "malo" de Guerrero, la biografía de Lafragua toma con pasión partido por el caudillo suriano. Más de la mitad del texto está dedicado a la aprehensión, juicio y fusilamiento de Guerrero. Resultan interesantes los juicios de un liberal sobre la independencia de México, dado que los liberales se asumen como hijos legítimos de sus principales protagonistas.

BCra Martínez Carvajal, Alejandro, Juan Escudero y Amadeo

Vidales, México, Editorial Revolución, 1961, 143 p.

Uno de los trabajos pioneros sobre el agrarismo y la organizacion de los trabajadores urbanos y agrícolas del estado de Guerrero. Se comienza por describir el puerto de Acapulco durante el Porfiriato y después se elaboran ląs biografias políticas de Escudero y Vidales, estrechamente ligadas con la historia de la costa guerrerense. Con dolor se narran sus trágicas muertes, a la vez que se rescata su aportación a la lucha de los trabajadores de la entidad.

\section{MEMORIAS}

BCRA

1934) del Partido Comunista Mexicano. Sin ser ficción, la obra está estructurada en forma de relato literario y a pesar de la tragedia que encierra -persecución, cárcel-el texto resulta ameno y de fácil lectura. A la autora se la considera una de las precursoras del feminismo en el estado de Guerrero.

BPCECH Martínez Carbajal, Alejandro, Memorias de la revolución s.c. en Guerrero, Acapulco, H. Ayuntamiento Constitucional, 1983,245 p., facs. y fotos.

El texto es una reproducción de las memorias del general Silvestre Mariscal elaborada por una de sus hijas. Mariscal, administrador de Correos al estallar la revolución de 1910, narra en sus memorias su participación en la lucha armada durante los años 1910-1917. Maderista y des- 
pués constitucionalista, Mariscal describe con detalle lo que fue la lucha contra los zapatistas y proporciona un panorama general sobre el proceso revolucionario en la Costa Grande del estado de Guerrero.

\section{GEOGRAFIA}

BPCECH Figueroa de Cotin, Esperanza, Atlas geografico e histórico s.c. del estado de Guerrero, Chilpancingo, FONAPAS Guerrero/Gobierno del estado de Guerrero, 1980, 171 p., ils. y fotos.

Trabajo apoyado en la documentación del Archivo de Estudios Geográficos e Históricos del Estado de Guerrero del Ing. Alejandro W. Paucic. El texto se compone de un atlas geográfico y otro histórico. El primero está formado por dos secciones: geografía física y geografia humana. La parte histórica informa sobre la conquista mexica del territorio del actual estado de Guerrero, la época colonial y la erección de la entidad en 1849. Incluye una bibliografía especializada y gran cantidad de mapas.

BPCECH García Rivas, Heriberto, Diccionario Guerrero. Enciclopes.c. dia regional, México, Fernández Editores, 1974, 224 p., ils. BNAL Breve diccionario que ofrece información sobre eco463 Dic. il Gue. nomía, división política y geografía -incluye un atlasde la entidad. Cuenta con un capítulo sobre la historia del estado así como con una sección de biografias. Tanto por su formato como por su contenido, este diccionario va dirigido especialmente a los estudiantes de nivel básico y de secundaria.

BPCECH González Dávila, Amado, Geografía del estado de Guerres.c. ro y síntesis histórica, México, Editorial Quetzalcóatl, $1959,140 \mathrm{p}$.

Amplia y documentada investigación sobre la historia y geografía del actual estado de Guerrero. El autor hace una breve narración de la historia de la entidad y explica las causas de su creación. En la parte propiamente geográfica se abordan los siguientes temas: orografía, hidrografia, clima, producción, agricultura, demografía, grupos humanos y comunicaciones. Sin duda, el texto de González Dávila es uno de los trabajos más completos sobre la materia.

BCRA López F., Héctor, Diccionario geográfico, histórico y G 977.7303

Lop. d lingulístico del estado de Guerrero, México, Editorial Pluma y Lápiz de México, 1942, 462 p., ils.

El autor, quien fuera gobernador del estado, comienza

BNAL

C

972.71003

Lop. d por hacer una breve reseña histórico-geográfica de la entidad, para después presentar el diccionario. En él se proporcionan datos sobre flora, lugares, nombres y personajes del estado. También se intercala información sobre hechos históricos ocurridos en la entidad. Al final se anexa un apéndice, el cual incluye biografías y monografias de los municipios guerrerenses.

BPCECH Luna Mayani, Raúl, Geografia moderna del estado de Gues.c. $\quad$ rrero, pról. de Angel Bassols Batalla, Ayutla de los Libres, Editorial Kotzaltzin, 1976, 326 p., fotos. 
El autor, economista de profesión, divide el texto en los siguientes temas: geografía física, geografía política, geografía humana, geografía económica y geografía regional. Sin afectar el rigor en la presentación de la información, el trabajo de Luna Mayani tiene como finalidad dirigirse a un público amplio, no especializado en la materia. Esta documentada investigación incluye numerosos mapas y una bibliografía.

BCRA Paucic, Alejandro W., Geografiageneral del estado de GueG17.273 rrero, S.l., FONAPAS Guerrero, 1980, 365 p., mapas.

Pau.g Obra póstuma del ingeniero yugoslavo muerto en 1980.

A lo largo de su vida, el autor formó el principal archivo sobre geografía, antropología, historia y otras materias con que cuenta el estado, el cual se encuentra bajo custodia del Instituto Guerrerense de la Cultura de Acapulco. El trabajo que comentamos da razón de las principales regiones de la entidad (Montaña, Tierra Caliente, Costa Grande y Costa Chica) y de sus recursos naturales. Esta obra representa tan sólo una pequeña muestra de la riqueza informativa que aún conserva el archivo Paucic, el cual, hasta el momento, ha sido poco estudiado.

BPFA Paucic, Alejandro W., Geografía histórica del estado de s.c. Guerrero, Acapulco, H. Ayuntamiento Constitucional, BIM

911.7273

Pau. $g$ 1980 , mapas.

Impresión de 35 mapas elaborados por Alejandro W.

Paucic y publicados con motivo de su muerte. El trabajo posee gran valor ya que, por ejemplo, en algunos mapas se da razón de las provincias tributarias de México-Tenochtitlan, en otros de las encomiendas y repartimientos que hubo en el actual estado de Guerrero durante la época colonial y, en otros más, de la división municipal al lograr Guerrero su conformación como entidad federativa.

BNAL Velasco, Alfonso Luis, Geografia y estadística del estado

917.2

Vel. $\mathrm{g} .7$

BCRA

G

Vel. $\mathbf{g}$ de Guerrero, México, Secretaría de Fomento, 1892 (Geografía y Estadística de la República Mexicana, 10).

Obra monumental escrita durante el porfiriato y dedicada a cada uno de los estados. El tomo X se ocupa de Guerrero. En él, después de describir la hidrografía, la orografía y el clima de la región, el autor hace un listado sumamente detallado de las riquezas naturales de la entidad, en especial, las vegetales. Proporciona el nombre vulgar y el científico de cada una de las plantas, agrupándolas con base en su uso y producción específicos. Al final de la obra, describe cada uno de los distritos que conforman el estado de Guerrero.

BNAL Viramontes, Leopoldo, Cartilla de geografía del estado de 908 Guerrero, 2a. ed, Chilpancingo, Imprenta del Gobierno en Mis. $65 \quad$ Palacio, 1893, 83 p.

Texto de divulgación en el cual se da razón de la historia y geografía de la entidad. El libro se encuentra organizado en forma de curso - dividido en lecciones - y aporta información sobre los distintos distritos que componen el estado, así como de las municipalidades que lo conforman. Al final del texto, el autor hace un recuento de los derechos y obligaciones de los ciudadanos guerrerenses. 


\section{ECONOMIA}

BPFA

s.c.

Alarcón Robledo, Sabás, Estado de Guerrero. Cuestiones económicas, México, Revista de Economía, 1967, 228 p., cdrs. estadísticos.

Se reúnen un conjunto de conferencias y artículos elaborados por el autor durante la década de los sesenta. El autor pretende esclarecer las causas del atraso económico del estado y, para ello, se aboca a estudiar la geografía, el elemento humano, lá economía y el desarrollo político de la entidad. Concluye que, dađa la riqueza natural de Guerrero, sus posibilidades de desarrollo son amplias. Incluye materiales estadísticos.

BPCECH Catalán Calvo, Rafael, Problemas de Guerrero, Chilpans.c. cingo, Gobierno del estado de Guerrero/Instituto Guerrerense de la Cultura, 1986, 172 p., ils.(Serie:Fuentes, 1).

Escrito en 1945, el texto presenta un diagnóstico de la situación del estado de Guerrero, realizado por quien fuera gobernador de la entidad durante el periodo 1941-1945. Los problemas económicos ocupan mayormente la atención del autor, para quien "...el factor principal para el mejoramiento económico de Guerrero es la construcción del ferrocarril Balsas-Acapulco [no realizada hasta la fecha] y de las carreteras de Tierra Caliente, Costa Grande y Costa Chica". Por tocar algunos problemas centrales del estado - educación, justicia, industria y agricultura - la lectura de este texto resulta útil.

BNAL Hernández García, Beatriz, Estado de Guerrero, México, 0821 SEP, 1968, 71 p., ils. (Serie Monografías de México. Cua-

Cl.P.131-135 dernos de Lectura Popular, 132).

Texto de divulgación sobre historia, geografía y economía de la entidad. La parte histórica da razón de los primeros asentamientos indígenas, de la Conquista, época colonial, Independencia, guerra de Reforma y Revolución. Se hace especial énfasis en la erección del estado, ocurrida en 1849. El capítulo dedicado a la geografía es bastante reducido, en tanto que el aspecto económico ocupa en mayor medida la atención de la autora. Al final se incluye una bibliografía básica.

вмсн Peña, Moisés T. de la, Guerrero económico, Chilpancingo, s.c. Gobierno del estado de Guerrero, 1949, 2 vols.

BPCECH Trabajo interdisciplinario en el que colaboraron un s.c. conjunto de especialistas (Jesús Martínez, Hilario Miramontes, José Arreola, Benjamín Retchkiman y Roberto Mejía). Se estudian de manera pormenorizada el medio físico, la población, la propiedad territorial, las comunicaciones, la ganadería, la silvicultura y pesca, la agricultura, la industria, el crédito, la hacienda pública, el comercio y el turismo. Sin duda, uno de los trabajos más completos sobre la economía guerrerense.

BCRA Retchkiman K., Benjamín, Recursosy problemas económiG30.97273 cos de la costa de Guerrero, México, s.e., 1948, 185 p., maRet. $\mathbf{r}$ pas.

Trabajo publicado en 1948 por el investigador de la UNAM recientemente fallecido. Se analizan los distintos aspectos de la economía de la costa guerrerense y se revisan con agudeza el sistema impositivo y la política fiscal, evaluando su influencia so- 
bre el conjunto de la economía costera. Después de hacer un diagnóstico sobre la economía regional, el autor realiza una serie de sugerencias y propuestas en materia de política económica.

BPCECH Torres, Manuel, Luis Méndez y Jorge Arcila, La estructus.c. ra económica del estado de Guerrero, Chilpancingo, Universidad Autónoma de Guerrero, 1983, 287 p.

Se analizan tres sectores de la economía guerrerense: el agrario, el manufacturero y los servicios. En los tres casos los autores comienzan por estudiar la relación de estos sectores con la economía nacional y después se evalúa su importancia dentro del contexto regional. Contiene numerosas estadísticas. Para la elaboración de la investigación, los autores contaron con la asesoría de Ugo Pipitone.

\section{ANTROPOLOGIA}

BIM

305.896

072

Agu. c

Aguirre Beltrán, Gonzalo, Cuijla: esbozo etnográfico de un pueblo negro, 2a. ed, México, Fondo de Cultura Económica, 1974, 242 p., ils,. mapas y retrs. (Sección de Obras de Antropología).

Clásico de la antropología mexicana, publicado por primera vez en 1958, apoyado en el trabajo de campo realizado en el pueblo negro de Cuajinicuilapa, Guerrero. En trabajos anteriores, el autor combatio el mito según el cual los pobladores negros sólo se asentaron en las costas demostrando cómo incursionaron tierra adentro, incorporándose-durante la época colonial-al trabajo en las minas y a los obrajes ubicados en las ciudades de mayor tamaño. Tomando como punto de partida sus anteriores hallazgos, Aguirre Beltrán emprendió el estudio de los pobladores negros de Cuajinicuilapa de la actualidad, vinculando el trabajo de campo con la indagación en el Archivo General de la Nación.

BPCECH Schmidt Schoenberg, Paul, et al., Arqueología y etnohistos.c. ria del estado de Guerrero, México, INAH/Gobierno del estado de Guerrero, 1986, $595 \mathrm{p}$.

Obra que reúne las ponencias presentadas en el Primer Coloquio de Arqueología y Etnohistoria del Estado de Guerrero, celebrado en noviembre de 1984 en la ciudad de Chilpancingo. Para el estudio de la historia reciente de la entidad resultan de interés los trabajos de Una Canger ("Los dialectos del náhuatl de Guerrero"), Gabriel Moedano Navarro ("Notas etnohistóricas sobre la población negra de la Costa Chica") y Juan Carlos Catalán Blanco ("El archivo Paucic en la reconstrucción de la historia del estado de Guerrero"), Desde el ángulo de la antropología, el conjunto de ponencias arroja luz sobre la historia y formación social guerrerenses, así como sobre los grupos étnicos asentados en la entidad.

\section{PROBLEMAS AGRARIOS}

BPCECH Gomezjara, Francisco A., Bonapartismo y lucha campesis. c. na en la Costa Grande de Guerrero, México, Editorial Posada, 1979, 323 p., fotos. (Colección Ideas Políticas).

Amplia y documentada investigación acerca de la relación entre los movimientos campesinos y el estado. Con dicha pretensión, el autor revisa la historia agraria de Guerrero desde la época prehispánica hasta el 
movimiento guerrillero dirigido por Genaro Vázquez Rojas. Se analizan los orígenes del caciquismo y la dinámica de las luchas agrarias encabezadas por Juan R. Escudero, Amadeo Vidales, Valente de la Cruz, Feliciano Radilla y María de la $O$. Pese a privilegiar el análisis sociologico, el trabajo se apoya en un buen conocimiento de la historia de la región. En 1977, Francisco A. Gomezjara obtuvo con esta obra el Premio Jose Revueltas, otorgado por la Editorial Posada.

BNAL Reina, Leticia, Las rebeliones campesinas en México 333.530972 (1819-1906), México, Siglo XXI Editores, 1980, 438 p., ils. Rei. r (Colección América Nuestra, 28).

Parte de la investigación está dedicada a las luchas campesinas en el actual estado de Guerrero, durante el periodo 1843-1849. El trabajo, fundamentalmente narrativo, da razón de las insurrecciones campesinas en la región de la Montaña - en especial de tlapanecos- en contra del despojo de sus tierras comunales perpetrado por los hacendados. En el capítulo dedicado a Guerrero, la autora (egresada de la Escuela Nacional de Antropología e Historia) se apoyó en fuentes documentales pertenecientes al Archivo de la Secretaría de la Defensa Nacional. En la solución de estos conflictos participo Juan Nepomuceno Alvarez; su papel mediador se aprecia con claridad en los documentos presentados por Leticia Reina.

BPCECH RÍos Morales, Manuel, Régimen capitalista e indígenas en s.c. La Montaña de Guerrero, Chilpancingo, Universidad Autónoma de Guerrero, 1983, 173 p. (Serie Historia de

Guerrero, 1).

Amplia investigación sobre las formas de producción predominantes en la región de la Montaña de Guerrero. El trabajo se desenvuelve en dos planos distintos: en el primero de ellos se analizan las características generales de la entidad (geográficas, demográficas y economicas) y las propias de la región de la Montaña, estas últimas acompañadas de un marco histórico. El segundo plano en que se desarrolla la investigación es el teorico-metodológico; aquí, el autor distingue entre las formas de producción capitalistas y las que no lo son, para después esclarecer los vínculos entre ambas. De la lectura del texto se desprende la tendencia creciente de las formas de producción no capitalistas a incorporarse al mercado capitalista.

\section{VIAJEROS Y CRONISTAS}

condumex Andrade, Vicente de Paúl, "Un viaje a Chilapa leído en la

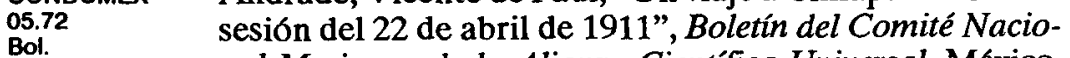
25933 nal Mexicano de la Alianza Científica Universal, México, núm. 10, junio de 1911, t. I, p. 304-307.

Vicente de Paúl Andrade no sólo describe Chilapa, sino también otros lugares como Chilpancingo, Tixtla, Tepechicotlán, Mochitlán, Tlamiztlahuacan y Quechultenango. En su relato se muestra estupor por la conclusión de la carretera de Iguala a Chilpancingo, que permitió hacer el recorrido entre las dos ciudades en un total de cuatro horas, empresa que anteriormente - sin poder hacer uso del automóvil-implicaba un viaje de dos días. Son valiosos los datos históricos, acerca de los poblados guerrerenses, esparcidos a lo largo del texto. 
BPCECH

S.c.

Benítez, José R., Guía histórica y descriptiva de la carretera México-Acapulco, México, Editorial Cultura, 1928, 198 p., fotos.

Este libro, escrito por un ingeniero, presenta, por un lado, una historia de los caminos en México desde la época colonial hasta 1927 y, por el otro, un conjunto de monografias sobre los puntos que toca la carretera México-Acapulco. La descripción detallada, el estilo ameno y la gran cantidad de lugares sobre los que brinda información, dan valor a este trabajo escrito por un "viajero" mexicano. El autor fue uno de los encargados de la construcción de la carretera, la Comisión Nacional de Caminos le encargó la elaboración de la guía.

BNAL

0821.1

Bep. 11-15

Calderón de la Barca, Frances Erskine Inglis de, La vida en México durante una residencia de dos años en ese país, pról. y trad. Felipe Teixidor, México, Editorial Porrúa, 1967, LXXI-428 p., fotos. (Sepan Cuantos, 74.)

Este comentado epistolario, escrito por la esposa del primer embajador de España en México, publicado por primera vez en 1843, presenta un verdadero mosaico de costumbres, lugares, vida política y social del México independiente. En uno de sus diversos viajes hacia el interior del país, la marquesa Calderón de la Barca visitó las grutas de Cacahuamilpa, quedando vivamente impresionada por sus imponentes formaciones. Desde distintos ángulos - literario, antropológico, etc.- este relato acerca de las grutas guerrerenses resulta interesante, aparte de su valor en tanto testimonio de un extranjero que visitó México.

BNAL Rivera Cambas, Manuel, México pintoresco, artístico y moG17.2 numental, México, Reforma, 1880-1883, 3 vols., ils.

Piv. $\mathrm{m}$

Una parte del tomo III de esta monumental obra está dedicada a varias de las localidades del estado de Guerrero. Se informa sobre Taxco, Tehuilotepec, Cacahuamilpa, Tlamacazapa, Iguala, Tuxpan, Tepecoacuilco, Mayanalan, Huitzuco, Cocula, Acatempan, Teloloapan, Tixtla, Chilapa, Tlapa, Chilpancingo, Ometepec, Xochistlahuaca, Cuajinicuilapa, Ayutla, Acapulco, Coyuca de Benítez, Tixtlancingo, San Marcos, Tecoanapa, El Coquillo, Cacahuatepec, Tecpan, Atoyac, San Jeronimo, Zihuatanejo, Coahuayutla, Zacatula, Ajuchitlán, Cutzamala y Coyuca de Catalán. Tanto por la información histórica como por las descripciones arquitectónicas esta obra resulta de gran interés.

BPCECH Verne, Julio, Un drama en México. Los primeros navios s.c. mexicanos, pról de Carlos Monsiváis, México, Editorial Hexágono, 78 p., ils.

Este relato, escrito hacia 1850 , narra el viaje imaginario de dos barcos españoles de la isla de Guam hacia Acapulco y de parte de la tripulación al interior de México. La narración está dividida en cinco jornadas: "De la isla de Guam a Acapulco", "De Acapulco a Pigualan", "De Pigualan a Taxco", "De Taxco a Cuernavaca" y "De Cuernavaca al Popocatépetl”. Viajero que nunca conoció México - como apunta Carlos Monsiváis en el prólogo-, Verne se apoyó en los trabajos de Pierre François de Pagès y Alexander von Humboldt para apuntalar algunas de las descripciones de su relato. Resultan de interés sus minuciosas observaciones sobre el clima, la topografía, la población y las costumbres de las distintas regiones del estado de Guerrero. 
COLMEX

910.4

Vicuña Mackenna, Benjamín, Páginas de mi diario duranV645p te tres años de viaje. 1853-1854-1855, Santiago, Universidad de Chile, 1936, 530 p., ils. (Obras Completas, 1).

Diario de viaje del expatriado chileno Benjamín Vicuña Mackenna. Al fracasar la rebelión de su padre, Pedro Félix Vicuña, el joven Benjamín de 22 años de edad, tiene que abandonar su país, dirigiéndose hacia América del Norte. A mediados de 1853 llega al puerto de Acapulco y emprende el trayecto hacia la ciudad de México. A su paso por el estado de Guerrero, va tomando nota de la geografía y costumbres de la región y establece un paralelismo con las de su país natal. Observador cuidadoso, se percata de la situación de pobreza en que viven las "clases bajas" guerrerenses.

\section{FOLKLORE}

BCRA s.c.

Altamirano, Ignacio Manuel, Paisajes y leyendas. Tradiciones y costumbres de México, Acapulco, H. Ayuntamiento Constitucional, 1983, $485 \mathrm{p}$.

Reunión de artículos publicados en distintos periodicos y tiempos por Altamirano y que fueron editados en conjunto durante 1884 . Se describen con detalle varias de las festividades religiosas (semana santa, día de muertos, etc.) que se realizan en México a lo largo del año. Un capítulo está dedicado a la vida en la ciudad de México y otro más a la celebración de la semana santa en su natal Tixtla.

BPMI Instituto Guerrerense de la Cultura, Calendario de fiestas s.c. del estado de Guerrero, Chilpancingo, Instituto GuerreBPCECH rense de la Cultura, 1987, 48 p., ils.

s.c. Primera aproximación a un calendario sobre las festividades de la entidad. En cada una de las fechas se consigna el nombre de la localidad donde se realiza la celebracion y se detallan algunas de sus características principales. Incluye tanto festividades civiles como religiosas.

BCRA Serrano Martínez, Celedonio, Coplas populares de GueG rrero, México, Editorial Libros de México, 1972, 334 p., ils.

Ser. c

Este texto constituye uno de los escasos estudios sobre el folklore de la región. Se brinda información sobre lo que es la copla, sus origenes y sus distintas temáticas. El autor clasifica, con base en su contenido, las principales coplas populares que se cantan en Guerrero e informa sobre algunos trovadores de la región.

\section{MONOGRAFIAS}

BPCECH Gutiérrez y Salgado, Angélica y Héctor Rodríguez Moras.c. les, Chilpancingo ayer y hoy, Chilpancingo, Instituto Guerrerense de la Cultura, 1987, 118 p., fotos.

Historia de la ciudad de Chilpancingo desde su fundación en 1591 hasta la actualidad. La lucha por la independencia y el Primer Congreso de Anáhuac ocupan la atención de los autores al estudiar el siglo XIX. Varias páginas se dedican a la revolución de 1910, en especial a la influencia del zapatismo en la región, y al paso de Alvaro Obregón por la ciudad en 1920. No obstante que el texto no profundiza en el análisis, su 
valor radica en la información que proporciona sobre la capital del estado y los hechos ligados a la misma. Es una de las pocas monografías sobre Chilpancingo. $\begin{array}{ll}\text { BCPA } & \text { Heredia Villarreal, J. de Jesús, Monografia de Arcelia, } \\ \mathrm{G} & \text { Gro., México, Ediciones e Impresiones Rodas, 1966, 244 } \\ \mathbf{9 7 2 . 7 3 8} & \text { p., fotos. }\end{array}$

Breve reseña histórica y descripción geográfica de este pueblo, ubicado en la región de Tierra Caliente de Guerrero. El autor se ocupa de la economía y de la acción estatal en la localidad, para después hacer mención de la obra realizada por el general Lázaro Cárdenas, en su calidad de vocal ejecutivo de la Comisión del Río Balsas. Se incluye un capítulo en el cual se narran algunos acontecimientos de la revolución de 1910 ocurridos en la región.

López Barroso, Epigmenio, Diccionario geográfico, S.c. histórico y estadístico del distrito de Abasolo, Guerrero. HeBotas, 1967, 340 p. chos históricos propios de esa región, México, Ediciones

El presente trabajo constituye uno de los pocos estudios acerca de la región conocida como Costa Chica del estado de Guerrero. No obstante llamarse "diccionario", el texto de hecho se inscribe en lo que Luis González ha denominado microhistoria. Además de proporcionar datos sobre los individuos más destacados de Ometepec, el libro informa sobre las costumbres y geografía regionales.

BPFA Sánchez Crispín, Alvaro, Relaciones espaciales entre Acas.c. pulco y su región, México, UNAM/Facultad de Filosofía y Letras, 1983, 311 p., mapas (tesis de maestría).

Amplio y documentado trabajo de tesis sobre el puerto de Acapulco. Se analiza la urbanización del puerto dentro del contexto nacional y se revisan las características geográficas de su entorno. En uno de los capítulos más relevantes se da una visión panorámica de la historia de Acapulco, desde los tiempos precolombinos hasta el siglo $\mathrm{XX}$, y después se muestran sus aspectos económicos sustantivos. Concluye la investigación con un análisis pormenorizado de la relación de Acapulco con el conjunto de la costa guerrerense. Incluye un listado bibliográfico y gran cantidad de mapas.

BPCECH Vidal Duarte, Francisco, San Luis Acatlán, Chilpancingo, s.c. Instituto Guerrerense de la Cultura, 1987, 75 p., ils.

Monografía del pueblo de San Luis Acatlán, cabecera del distrito de Altamirano a partir del año de 1944. El trabajo da razón de la primera villa fundada por los españoles en la costa del Pacífico y de la resistencia de los indios yopes a la conquista, de las migraciones indígenas y de las calamidades y epidemias sufridas por la población durante el siglo XIX y principios del XX. Narra un episodio de la revolución de 1910 verificado en aquel poblado. Vidal Duarte, michoacano residente en Chilpancingo, le da un carácter fundamentalmente descriptivo a su monografía. 


\section{LITERATURA}

BNAL

M863.4

Poni. H

1970

Poniatowska, Elena, Hasta no verte Jesús mío, México, Ediciones Era, 1969, 316 p.

En esta novela, basada en el testimonio de Jesusa Pa-

lancares, se recrean los años de la Revolución mexicana en distintas partesdel país. Jesusa Palancares, oriunda de Oaxaca, colaboró con el ejército constitucionalista, participando en algunas acciones militares en el estado de Guerrero. La descripción de lo que significo para las masas populares (integrantes del bloque de los "revolucionados" al decir de Luis González) la lucha armada y el relato minucioso de la vida cotidiana en Guerrero y en otras regiones del país resulta de gran interés. Sin duda, a través de la pluma de Elena Poniatowska se expresa un valioso testimonio sobre la historia de Guerrero y del país.

BPCECH Vidal Duarte, Francisco (comp.), Cuentos fantásticos y s.c. campiranos en Guerrero, Chilpancingo, Instituto Guerrerense de la Cultura, 1984, 196 p., ils. (Serie de Rescate, 1).

Compilación de 38 cuentos populares, rescatados por distintos investigadores, provenientes de las cuatro regiones del estado de Guerrero (Montaña, Tierra Caliente, Costa Grande y Costa Chica). Muchos de estos cuentos son adaptaciones "regionales" de famosos y conocidos cuentos europeos.

\section{LEGISLACION}

BNAL Constituciones, Constitución Política del Estado Libre y 308 Soberano de Guerrero, sancionada por su H. Legislatura el Misc. 121 de noviembre de 1880, Chilpancingo, Gobierno del estado de Guerrero, 1893, 48 p.

Texto de la Constitución de 1880 que incorpora las modificaciones sufridas por la misma a lo largo de una década. En 1884, bajo la gubernatura de Diego Alvarez, el VIII Congreso Constitucional introdujo cambios en el texto de la carta magna. Posteriormente, durante la gubernatura de Francisco $O$. Arce, se reformó el artículo 18, referente a la calificación de elecciones. En 1890 se modificó una vez más la Constitución guerrerense.

CONDUMEX Leyes, decretos, etc., Ley orgánica provisional para el arre972.71 glo interior del estado de Guerrero, México, Imprenta de Ig-

A.N 25078-C nacio Cumplido, $1850,55 \mathrm{p}$.

Fue promulgada en Iguala de Iturbide el 15 de marzo

de 1850, siendo gobernador constitucional del estado de Guerrero el general Juan N. Alvarez. Esta ley fijo las funciones, responsabilidades y atribuciones de los funcionarios adscritos a los poderes Ejecutivo y Judicial. Por otra parte, reconoce a la entidad guerrerense como integrante de la Federación Mexicana y faculta “... al gobierno para que los empleos de que habla esta ley los provea según lo permitan las circunstancias del erario y necesidades de los distritos".

CONDUMEX Leyes, decretos, etc., Ley de administración política y mu353.272.71 nicipal de los distritos del estado, Chilpancingo, Tipografía 
de los pueblos corre a cargo de los prefectos; ayuntamientos, comisarios y subcomisarios. El prefecto cumpliría la función de intermediario entre el gobierno estatal y el municipal (a cargo del ayuntamiento). Por su parte, comisarios y subcomisarios de policía ejercerían - según esta ley- la administración municipal en los pueblos que carecieran de ayuntamiento.

CONDUMEX Leyes, decretos, etc., Ley de hacienda del estado, Chilpan353.271.71 cingo, Tipografía del Gobierno del estado de Guerrero, Gue. A.N 907-c $1882,16 \mathrm{p}$.

Expedida en Chilpancingo de los Bravos en octubre de 1882, siendo gobernador de la entidad el general Diego Alvarez. Estableció, entre otras cosas, que todo residente (varón) en el estado de Guerrero que tuviera 18 a 60 años de edad pagaría mensualmente 12 centavos de contribución personal.

CONDUMEX Leyes, decretos, etc., Ley orgánica electoral para el nom353.272.71 bramiento de individuos de los supremos poderes LegislatiA.N 907-c vo y Ejecutivo y demás empleados que son de elección popular en el estado de Guerrero, Chilpancingo, Tipografía del Gobierno del estado de Guerrero, $1883,19 \mathrm{p}$.

Expedida en Chilpancingo de los Bravos en octubre de 1882. Establece que son cargos de elección popular en la entidad los de: gobernador, diputado, jueces menores, regidor y síndico. Por otra parte, determina que los cargos de comisario y subcomisario de policía, así como los de inspector y subinspector, serán otorgados por la propia corporación policiaca. Concede el derecho al voto a todos los ciudadanos mayores de 21 años o de 18 en el caso de estar casados.

CONDUMEX Leyes, decretos, etc., Reglamento interior del Superior Tri353.272.71 bunal de Justicia del estado de Guerrero, Chilpancingo, TiGue. $\quad$ pografía del Gobierno del estado de Guerrero, 1883, 4 p.

Expedido en octubre de 1882 en la ciudad de Chilpancingo. Establece, entre otras cuestiones, las atribuciones de cada una de las salas del tribunal, los requisitos para ser nombrado juez y las funciones y obligaciones del abogado de pobres (defensor de oficio).

\section{BIBLIOGRAFIAS Y GUIAS DOCUMENTALES}

BPCECH Centro de Investigaciones Sociales, Bibliografia Guerrero. s.c. $\quad 500$ fuentes para el estudio económico y social del estado, Chilpancingo, Universidad Autónoma de Guerrero, $1975,111 \mathrm{p}$.

Trabajo elaborado por un equipo de investigadores y alumnos de la Universidad Autónoma de Guerrero. Reúne fuentes bibliográficas y hemerográficas sobre historia, economía y sociedad en Guerrero durante los siglos XIX y XX, aunque también consigna algunos trabajos referidos a la época colonial. El enlistado de textos incluye desde obras generales sobre el país y la entidad hasta estudios específicos sobre las distintas regiones guerrerenses. Esta investigación bibliográfica es una de las pioneras en la entidad. 
Las guías fueron elaboradas con base en los acervos documentales del Archivo General de la Nación (AGN), Archivo Histórico de la UNAM (AH/UNAM), Centro de Estudios sobre la Universidad (CESU), Centro de Estudios de Historia de México (CEHM), Archivo Amadeo Vidales (AV) y Coleccion Lafragua de la Biblioteca Nacional (COL.LAF). Las ocho que componen esta colección se refieren a: I. Archivo Amadeo Vidales (ubicado en Acapulco y perteneciente a la familia Vidales); II. Fondo Francisco I. Madero (AGN); III. Ramo Bienes Nacionalizados (AGN); IV. Archivo Genovevo de la O (AGN); V. Colección Lafragua (BN); VI. Archivo Gildardo Magaña (CESU); VII. Ramo Gobernación (AGN), y VIII. Fondo Emiliano Zapata (AGN). Los documentos están clasificados cronologicamente y todos se refieren al estado de Guerrero.

BPCECH Marino Flores, Anselmo, Juan Carlos Catalán Blanco s.c. y Roberto Cervantes Delgado, Bibliografia ántropológica del estado de Guerrero, Chilpancingo, Gobierno del estado de Guerrero/Instituto Guerrerense de la Cultura, 1987, 328 p., mapas y fotos. (Serie Fuentes, 3.)

Los autores presentan una bibliografia comentada compuesta por 940 textos, organizados en ocho secciones: I. Obras generales; II. Antropología social y etnografia; III. Arqueología, prehistoria y arte; IV. Etnohistoria; V. Linguística; VI. Folklore y narrativa indígena; VII. Antropología física, y VIII. Miscelánea. En especial, las secciones I, IV y VIII consignan obras referidas a la historia, geografía y vida social de la entidad. No obstante su brevedad, los comentarios a cada uno de los libros dan razón, por lo general, del contenido de los textos. Sin duda, este trabajo constituye la recopilación bibliográfica más amplia y sistemática sobre el estado de Guerrero.

BNAL $\quad$ Mora.

COLMEX Biblioteca "Daniel Cosío Villegas" de El Colegio de México.

CONDUMEX Biblioteca del Centro de Estudios de Historia de México.

INAH

BCRA

BPFA Biblioteca Instituto Nacional de Antropología e Historia. Biblioteca "Carmen Romano de López Portillo". Acapulco.

$\mathrm{BMCH}$

$\mathrm{BPCECH}$ Biblioteca Pública Federal "Dr. Alfonso S. Alarcón". Acapulco. Biblioteca Municipal "Marfa Luisa Ocampo Heredia". Chilpancingo.

BCUAG Biblioteca Central de la Universidad Autónoma de Guerrero. Chilpancingo.

BEEUAG Biblioteca de la Escuela Superior de Ciencias Económicas de la Universidad Autónoma de Guerrero. Chilpancingo.

BPMI Biblioteca Pública Municipal Alameda "Ambrosio Figueroa" Iguala. 